\title{
Reconsideration of indications for choledochoscopic gallbladder-preserving surgery and preventive measures for postoperative recurrence of gallstones
}

\author{
Qian Cheng $\mathrm{Du}^{1}$, Yan Yan Wang ${ }^{2}$, Chen Liang $\mathrm{Hu}^{3}$, Ying Zhou ${ }^{3}$ \\ ${ }^{1}$ Department of General Surgery, Shanghai Fourth People's Hospital Affiliated to Tongji University School of Medicine, Shanghai, China \\ ${ }^{2}$ Department of Hematology, Affiliated Fuyang Hospital of Anhui Medical University, Fuyang, China \\ ${ }^{3}$ Department of Hepatopancreatobiliary Surgery, the Affiliated Hospital of Qinghai University, Xining, China
}

Videosurgery Miniinv 2020; 15 (1): 87-96 DOI: https://doi.org/10.5114/wiitm.2019.88647

\begin{abstract}
Introduction: The recurrence of cholecystolithiasis after choledochoscopic gallbladder-preserving surgery is a major problem. It is unclear whether the recurrence of cholecystolithiasis is due to the limitations of the operation itself or to the selection of inappropriate candidates.

Aim: To analyze the recurrence of cholecystolithiasis after choledochoscopic gallbladder-preserving surgery and to provide guidance for the treatment of cholecystolithiasis by choledochoscopic gallbladder-preserving surgery.

Material and methods: A total of 145 patients who had undergone choledochoscopic gallbladder-preserving surgery were studied from January 2012 to January 2018. The recurrence rate, recurrence time, and risk factors were evaluated.

Results: Of these 145 patients, 14 (9.66\%) experienced recurrence with a mean follow-up time of $39.72 \pm 24.44$ months. The mean time to recurrence was $30.07 \pm 21.21$ months. Univariate analysis showed that pregnancy histo$r y(p=0.008)$, the uniformity of gallstones $(p=0.002)$, preoperative inflammation $(p=0.022)$, postoperative oral drugs $(p=0.022)$ and the regularity of diet $(p=0.001)$ were significantly related to recurrence. The uniformity of gallstones (odds ratio $(O R)=0.079 ; 95 \%$ confidence interval $(C I): 0.010-0.590 ; p=0.013$ ) and the regularity of diet $(O R=0.074 ; 95 \% \mathrm{Cl}: 0.010-0.528 ; p=0.009)$ were independent prognostic factors for recurrence according to multivariate analysis.

Conclusions: Nonuniform gallstones combined with an irregular diet are significant risk factors that predict cholecystolithiasis recurrence.
\end{abstract}

Key words: cholecystolithiasis, gallbladder preservation, recurrence, risk factors.

\section{Introduction}

Cholecystolithiasis is a common digestive system disease worldwide, and the prevalence is estimated to be 10-20\% [1]. Laparoscopic cholecystectomy (LC) is the main treatment method for cholecystolithiasis, and this procedure has been regarded as the 'gold standard' approach for several years. How- ever, postcholecystectomy syndrome, which includes chronic abdominal discomfort, alkaline reflux gastritis, dyspepsia, fatty diarrhea, bile duct injury, the occurrence of colonic cancer and other symptoms, has been reported by previous experimental and clinical studies [2-5]. With the deeper recognition of gallbladder function, many hepatobiliary surgeons

Address for correspondence

Prof. Ying Zhou, Department of Hepatopancreatobiliary Surgery, The Affiliated Hospital of Qinghai University, Qinghai University, No. 27 Tongren Road, 810000 Xining, China, phone: +86 971 6230701, e-mail: 17697227511@163.com 
argue that cholecystectomy for gallstones presents the possibility of unnecessary excision or overtreatment.

Choledochoscopic gallbladder-preserving surgery is a minimally invasive surgical method to remove gallstones and retain the function of the gallbladder. Percutaneous cholecystolithotomy was first described as a treatment for gallstones in 1985, and the indications were for patients with related symptoms or who demonstrated a serious risk of LC [6]. Cholecystolithotomy is suitable for almost $78 \%$ of patients with gallstones, but gallstone recurrence after the operation occurs in $31 \%$ to $44 \%$ of these patients [7]. Therefore, the development of gallbladder-preserving therapies has been stalled. The recurrence rate of gallstones has been significantly reduced by the development of minimally invasive techniques, the recognition of surgical indications and effective postoperative preventive measures [8, 9]. However, it is still unclear which possible factors could be used to predict the recurrence of cholecystolithiasis after choledochoscopic gallbladder-preserving surgery.

Regular diet can reduce the cholesterol saturation of bile in the gallbladder and can decrease the risk of cholestasis from regular gallbladder emptying $[10,11]$. The uniformity of gallstones is an important factor affecting the rate of stone clearance by shockwave lithotripsy [12]. However, there have not been any other reports related to the regularity of diet and the uniformity of gallstones in terms of recurrence factors or the time to recurrence of cholecystolithiasis. To better evaluate the feasibility of choledochoscopic gallbladder-preserving surgery, it is necessary to analyze the possible factors associated with recurrence and the time to recurrence.

\section{Aim}

The aims of the present study were to predict the recurrence rate with a long-term follow-up, analyze the factors associated with gallstone recurrence and select suitable candidates in order to prevent the recurrence of gallstones after choledochoscopic gallbladder-preserving surgery.

\section{Material and methods}

The protocol was approved by the Institutional Review Board of Qinghai Renji Hospital and was successfully registered in the Chinese Clinical Trial Registry (ChiCTR, No. ChiCTR1900020779).

\section{Patients}

A retrospective medical chart review was conducted for patients who had received choledochoscopic gallbladder-preserving surgery at the affiliated hospital of Qinghai University and at Qinghai Renji Hospital from January 2012 to January 2018. The diagnostic criteria for cholecystolithiasis are based on color Doppler ultrasound. Gallstones appear as mobile hyperechoic foci that cast marked posterior acoustic shadowing [13]. Patients who were diagnosed with cholecystolithiasis and who received choledochoscopic gallbladder-preserving surgery were enrolled in this study. Patients without postoperative follow-up data or who could not be followed up and who had a postoperative follow-up time of less than 6 months were excluded.

\section{Clinical data}

Baseline data, including sex, age, body mass index (BMI), pregnancy history, family history, diet, blood type, preoperative symptoms and neutrophil ratio as well as preoperative alanine transaminase (ALT), preoperative aspartate amino transferase (AST), postoperative ALT, postoperative AST, preoperative total cholesterol (TCH), preoperative triglyceride (TG), preoperative high-density lipoprotein $(\mathrm{HDL})$, preoperative low-density lipoprotein (LDL), preoperative apolipoprotein A (APOA), and preoperative apolipoprotein B (APOB) levels, were collected. The follow-up visit was recorded, including the date and ultrasound results. The uniformity of gallstones was graded as uniform (solitary gallbladder stone or the size and shape of all gallstones were approximately the same) or nonuniform (the size and shape of multiple existing gallstones were different). The regularity of diet was graded as regular (not skipping breakfast or reducing the interval between two meals) or irregular (not eating breakfast, eating one meal and forgetting the next meal, or a long interval between two meals) [14]. The recurrence of cholecystolithiasis was defined as no residual gallstones found in the gallbladder during the operation and postoperative color Doppler ultrasound revealing gallstones in the gallbladder.

\section{Treatment}

The informed consent form for surgery was signed by all patients or authorized trustees. Most patients 
underwent choledochoscopic gallbladder-preserving surgery, and a few patients underwent laparoscopic choledochoscopic gallbladder-preserving surgery. We selected an incision under the right costal margin, and the length of the incision was approximately 4 centimeters under general anesthesia. The fundus of the gallbladder was found, and it was guided toward the incision (part of the operation was assisted by laparoscopic monitoring). A small incision was made at the bottom of the fundus of the
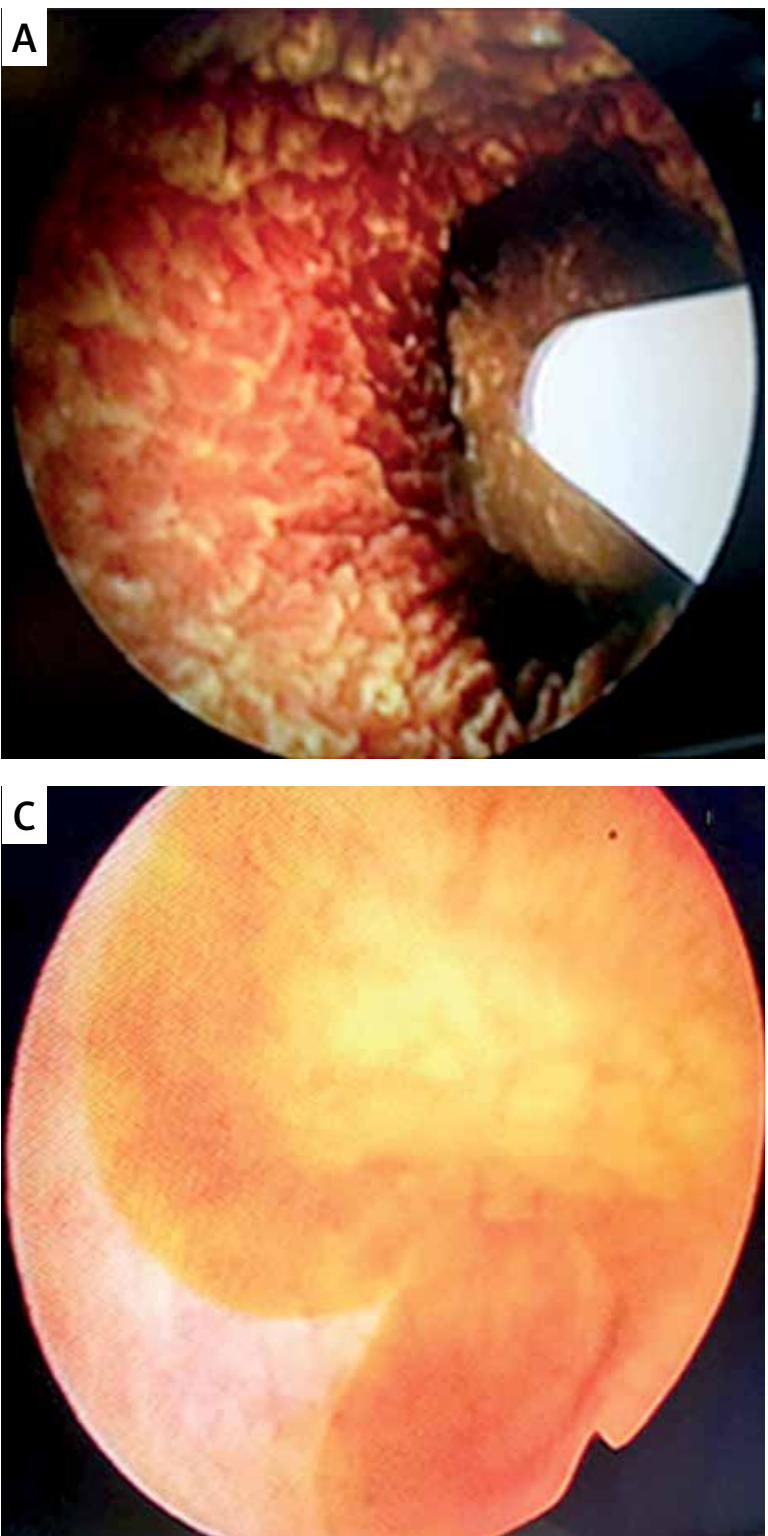

Photo 1. Surgery photographs. A - The gallbladder stone was clearly seen after the bile was suctioned out, $\mathbf{B}$ - the gallstones were removed from the gallbladder using a grasping basket guided by the soft fiber choledochoscope, $\mathbf{C}$ - the bile was observed to flow from the neck of the gallbladder into the fundus of the gallbladder, D - using stitches the fundus of gallbladder was sutured continuously
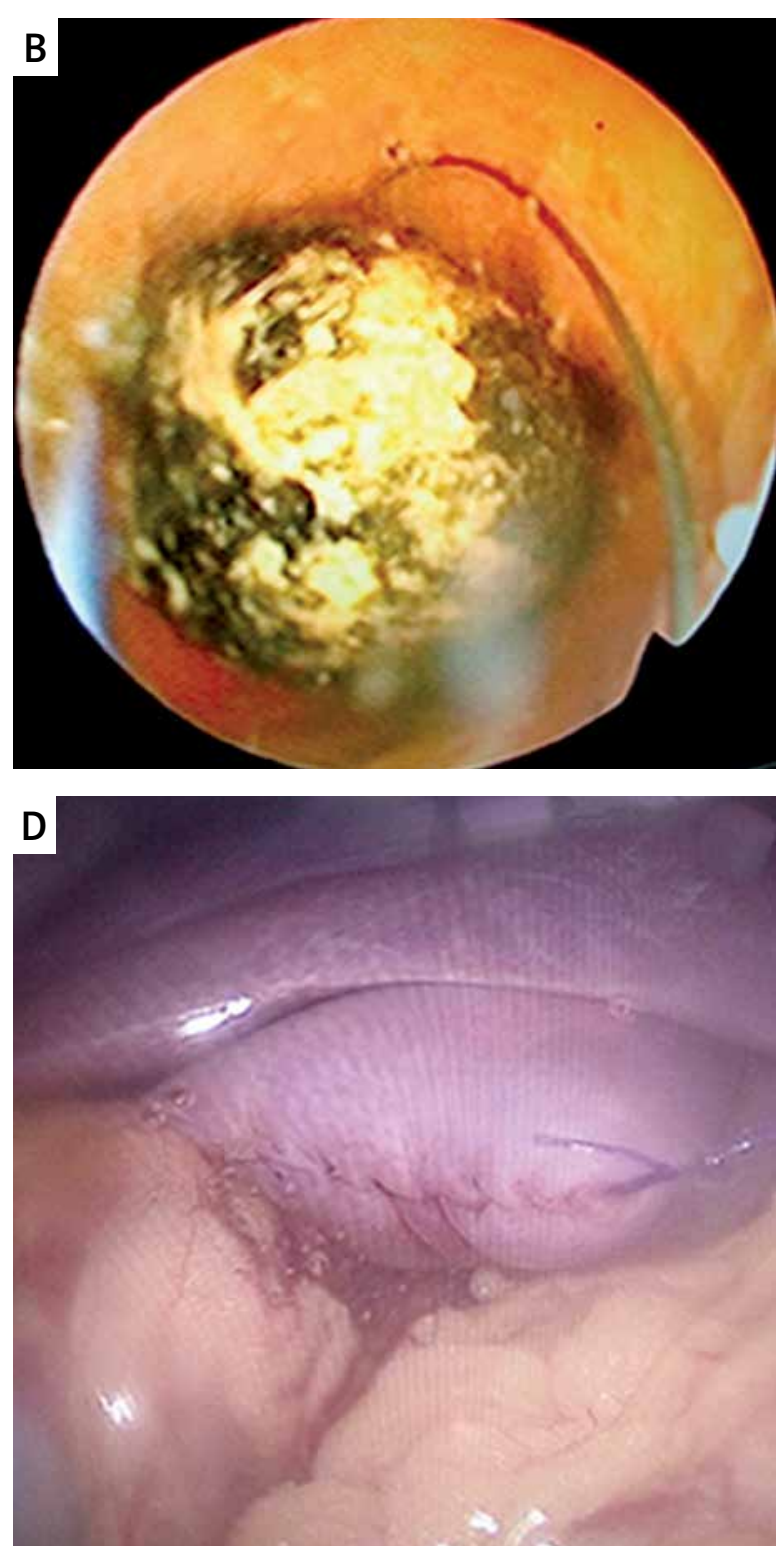

gallbladder according to the size of the stone. After the bile was suctioned out, a soft fiber choledochoscope was placed into the gallbladder (Photo $1 \mathrm{~A}$ ). The gallstones were removed from the gallbladder using a grasping basket guided by the soft fiber choledochoscope (Photo $1 \mathrm{~B}$ ). When the gallstones were removed completely, the choledochoscope was placed into the gallbladder again. The bile was observed to flow from the neck of the gallbladder into the fundus of the gallbladder because the neck of the

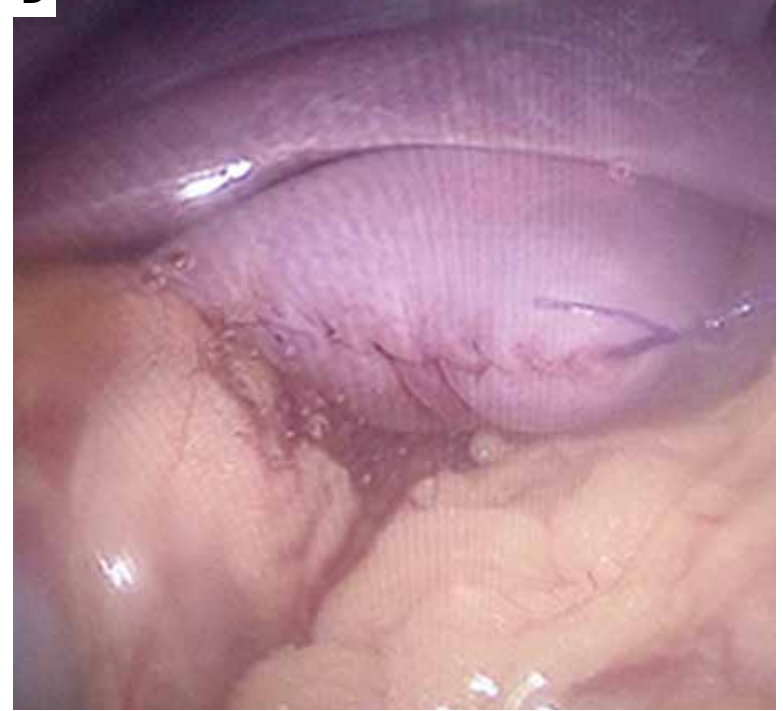


gallbladder did not have residual stones (Photo $1 \mathrm{C}$ ); then, using stitches, the fundus of the gallbladder was sutured continuously (4/0 Vicryl Plus) (Photo 1 D). In the absence of active bleeding and bile leakage, the drainage tube was not routinely placed in the surgical field.

\section{Postoperative management}

The patients usually resumed a normal diet after $8 \mathrm{~h}$ of postoperative fasting. All patients were discharged from the hospital 2 days after surgery. Postoperative oral ursodeoxycholic acid (5 mg/kg/ day) was administered for 3 months routinely, and patients were encouraged to eat fatty meals regularly after surgery to promote regular gallbladder contractions. Ultrasound examination was performed approximately every 6-12 months after the operation. If ultrasonography revealed gallstones in the gallbladder, the time of recurrence of gallstones was recorded.

\section{Statistical analysis}

Statistical analysis of the data was performed using SPSS software version 23.0 (IBM Corporation, 2015, USA). All continuous variables that followed a normal distribution are described as the mean \pm standard deviation and were compared using the independent $t$-test. The univariate analysis of the postoperative recurrence and possible clinical factors was tested by Pearson $\chi^{2}$ test. Recurrence-free survival was analyzed using Kaplan-Meier curves with statistical comparison with the log-rank test. A logistic regression analysis model was established to assess the relationship between postoperative gallstone recurrence and possible clinical factors. A bilateral probability $(p)$ value $<0.05$ was considered statistically significant.

\section{Results}

A total of 145 patients with cholecystolithiasis were treated with choledochoscopic gallbladder-preserving surgery. The postoperative complications of the patients were evaluated by the Clavien-Dindo classification, and all patients were considered grade I. The mean age of these patients was $36.60 \pm 11.57$ years with an age range of 14-52 years. The mean follow-up time was $39.72 \pm 24.44$ months, ranging from 6 to 90 months. The baseline characteristics of the included patients with choledochoscopic gallbladder-preserving surgery are listed in Table I.

After a median follow-up of 36 months, 14 out of 145 patients (9.66\%) experienced recurrence. Of the 14 patients who experienced recurrence, 10 (71.4\%) received cholecystectomy, $2(14.3 \%)$ received choledochoscopic gallbladder-preserving surgery, and $2(14.3 \%)$ received conservative treatment. The mean time to recurrence was $30.07 \pm 21.21$ months, ranging from 9 to 61 months after choledochoscopic gallbladder-preserving surgery. The estimated 1-year, 3-year and 5-year recurrence rates for all patients were $2.4 \%, 7.8 \%$ and $12.5 \%$, respectively (Figure 1 ).

Univariate analysis demonstrated that pregnancy history ( $p=0.008$, Pearson $\chi^{2}$ test), the uniformity of gallstones ( $p=0.002$, Pearson $\chi^{2}$ test), whether patients had preoperative inflammation $(p=0.022$, Pearson $\chi^{2}$ test), whether patients were administered postoperative oral drugs ( $p=0.022$, Pearson $\chi^{2}$ test) and the regularity of diet ( $p=0.001$, Pearson $\chi^{2}$ test) were factors that were significantly related to recurrence (Table II). Multivariate analysis according to the logistic regression model showed that the uniformity of gallstones $(\mathrm{OR}=0.079 ; 95 \% \mathrm{Cl}: 0.010-0.590 ; p=$ $0.013)$ and the regularity of $\operatorname{diet}(\mathrm{OR}=0.074 ; 95 \% \mathrm{Cl}$ : $0.010-0.528 ; p=0.009$ ) were independent prognostic factors for predicting recurrence (Table III).

The recurrence rates were analyzed using Kaplan-Meier curves, and the differences between patients with various clinical factors after choledochoscopic gallbladder-preserving surgery were assessed using the two-sided log-rank test. The estimated 1 -year recurrence rate of patients with uniform gallstones was $1.3 \%$ compared with $4.3 \%$ in patients with nonuniform gallstones. After 3 and 5 years, the recurrence rates were 4 versus $14 \%$ and 6.8 versus $22.4 \%$, respectively ( $p=0.005$, Figure 2 A). The estimated 1-year recurrence rate of patients with a regular diet was $1.1 \%$ compared with $5.7 \%$ in patients with an irregular diet. After 3 and 5 years, the recurrence rates were 4.8 versus $16 \%$ and 7.4 versus $24.2 \%$, respectively ( $p=0.002$, Figure $2 \mathrm{~B}$ ). Other factors, including age, sex, BMI, family history, smoking, long-term drinking, preoperative $\mathrm{TCH}$, preoperative $\mathrm{TG}$, the number of gallstones, the maximal diameter of gallstones, and gallbladder contraction function, were not significantly associated with recurrence.

The prognostic evaluation was established by counting the number of independent risk factors (nonuniform gallstones and an irregular diet) for 
Table I. Baseline characteristics of the included patients after gallbladder-preserving cholecystolithotomy

\begin{tabular}{|c|c|c|c|}
\hline \multirow[t]{2}{*}{ Variable } & \multirow[t]{2}{*}{ Total } & \multicolumn{2}{|c|}{ Recurrence } \\
\hline & & Yes & No \\
\hline Age [years]: & $36.60 \pm 11.57$ & & \\
\hline$>40$ & $67(46.2 \%)$ & 7 & 60 \\
\hline$\leq 40$ & $78(53.8 \%)$ & 7 & 71 \\
\hline \multicolumn{4}{|l|}{ Sex: } \\
\hline Female & $92(63.4 \%)$ & 10 & 82 \\
\hline Male & $53(36.6 \%)$ & 4 & 49 \\
\hline $\mathrm{BMI}\left[\mathrm{kg} / \mathrm{m}^{2}\right]:$ & $23.16 \pm 3.54$ & & \\
\hline$>28$ & $14(9.7 \%)$ & 3 & 11 \\
\hline$\leq 28$ & $131(90.3 \%)$ & 13 & 118 \\
\hline \multicolumn{4}{|c|}{ Pregnancy history (N): } \\
\hline$\geq 2$ & $56(68.3 \%)$ & 7 & 59 \\
\hline$\leq 1$ & $26(31.7 \%)$ & 3 & 23 \\
\hline \multicolumn{4}{|l|}{ Family history: } \\
\hline Present & $26(17.9 \%)$ & 5 & 21 \\
\hline Absent & $119(82.1 \%)$ & 9 & 110 \\
\hline \multicolumn{4}{|l|}{ Lifestyle: } \\
\hline Free & $40(27.6 \%)$ & 9 & 31 \\
\hline Regular & 105 (72.4\%) & 5 & 100 \\
\hline \multicolumn{4}{|c|}{ Preoperative symptoms: } \\
\hline Present & $120(82.8 \%)$ & 13 & 107 \\
\hline Absent & $25(17.2 \%)$ & 1 & 24 \\
\hline \multicolumn{4}{|c|}{ Number of gallstones: } \\
\hline$<3$ & $104(71.7 \%)$ & 7 & 97 \\
\hline$\geq 3$ & $41(28.3 \%)$ & 7 & 34 \\
\hline \multicolumn{4}{|c|}{ Maximal diameter of gallstones: } \\
\hline$<2 \mathrm{~cm}$ & $92(63.4 \%)$ & 6 & 86 \\
\hline$\geq 2 \mathrm{~cm}$ & $53(36.6 \%)$ & 8 & 45 \\
\hline \multicolumn{4}{|c|}{ Shape of gallstones: } \\
\hline Uniform & $96(66.2 \%)$ & 4 & 92 \\
\hline Non-uniform & $49(33.8 \%)$ & 10 & 39 \\
\hline \multicolumn{4}{|l|}{ Gallbladder wall: } \\
\hline Normal & 105 (72.4\%) & 12 & 93 \\
\hline Thickening & $40(27.6 \%)$ & 2 & 38 \\
\hline
\end{tabular}


Table I. Cont.

\begin{tabular}{|c|c|c|c|}
\hline \multirow[t]{2}{*}{ Variable } & \multirow[t]{2}{*}{ Total } & \multicolumn{2}{|c|}{ Recurrence } \\
\hline & & Yes & No \\
\hline \multicolumn{4}{|l|}{ Gallbladder mucosa: } \\
\hline Smooth & $112(77.2 \%)$ & 11 & 101 \\
\hline Rough & $33(22.8 \%)$ & 3 & 30 \\
\hline \multicolumn{4}{|c|}{ Gallbladder contraction function: } \\
\hline$<30 \%$ & $30(20.7 \%)$ & 4 & 26 \\
\hline$\geq 30 \%$ & $115(79.3 \%)$ & 10 & 105 \\
\hline \multicolumn{4}{|l|}{ Preoperative inflammation: } \\
\hline Present & $44(30.3 \%)$ & 8 & 36 \\
\hline Absent & $101(69.7 \%)$ & 6 & 95 \\
\hline White blood cells $\left[\times 10^{9} / 1\right]$ & $5.96(3.00-10.93)$ & $6.67(4.63-9.61)$ & $5.93(3.00-10.93)$ \\
\hline Neutrophil ratio (\%) & $56.70(36.20-80.90)$ & $56.62(48.20-77.30)$ & $56.70(36.20-80.90)$ \\
\hline Preoperative ALT [U/I] & $26(9-376)$ & $21(10-114)$ & $26(9-376)$ \\
\hline Preoperative AST [U/I] & $24(13-188)$ & $21.50(13-56)$ & $25(13-188)$ \\
\hline Postoperative ALT [U/I] & $23(12-117)$ & $19(16-117)$ & $23(12-78)$ \\
\hline Postoperative AST [U/I] & $25(15-77)$ & $26(19-43)$ & $25(15-77)$ \\
\hline Preoperative TCH [mmol/l] & $3.94(1.18-7.37)$ & $3.66(2.53-5.34)$ & $4.06(1.18-7.37)$ \\
\hline Preoperative TG [mmol/l] & $1.41(0.37-4.73)$ & $1.29(0.67-2.48)$ & $1.44(0.37-4.73)$ \\
\hline Preoperative HDL [mmol/ll] & $1.07(0.60-2.13)$ & $1.05(0.84-1.26)$ & $1.08(0.60-2.13)$ \\
\hline Preoperative LDL [mmol/l] & $2.30(1.02-4.36)$ & $2.07(1.20-3.04)$ & $2.32(1.02-4.36)$ \\
\hline Preoperative APOA [g/l] & $1.11(0.60-2.01)$ & $1.11(0.80-1.43)$ & $1.11(0.60-2.01)$ \\
\hline Preoperative APOB [g/l] & $0.74(0.26-1.91)$ & $0.80(0.26-1.91)$ & $0.74(0.36-1.23)$ \\
\hline
\end{tabular}

$T G$-triglyceride, $T C H$ - total cholesterol, $B M I$ - body mass index, $A L T$ - alanine transaminase, AST - aspartate amino transferase, HDL - high-density lipoprotein, $L D H$ - low-density lipoprotein, APOA - apolipoprotein A, APOB - apolipoprotein $B$.

each patient. The sum of the risk factors of each patient was calculated, and the final result is shown. All patients were divided into three levels based on the final results $(0,1$ and 2$)$. The frequency distribution of patients with different independent risk factors is shown in Figure 3. The greater the number of independent risk factors was, the higher was the recurrence rate.

\section{Discussion}

The treatment of cholecystolithiasis ranges from conservative treatment to resection surgery [1518]. Operative treatment still remains the first-line choice of treatment for cholecystolithiasis [19]. The two major types of operative treatment for cholecystolithiasis are cholecystectomy and choledochoscopic gallbladder-preserving surgery [20-22]. In our study, all patients with cholecystolithiasis underwent choledochoscopic gallbladder-preserving surgery, and this operation has been demonstrated to be a safe and effective operative method. In comparison with the benefits of cholecystectomy, those of choledochoscopic gallbladder-preserving surgery are significant, including fewer complications, fewer choledochus injuries and rapid recovery as well as rarely affecting digestive function [17, 23-25].

Although choledochoscopic gallbladder-preserving surgery has been indicated to successfully re- 


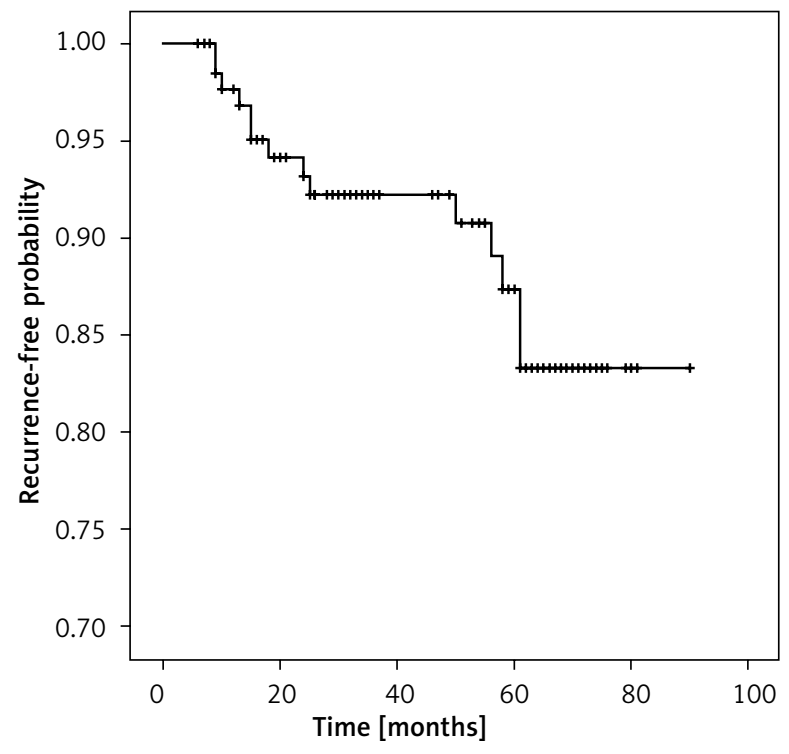

Figure 1. Kaplan-Meier survival curve of the estimated recurrence rate of 145 patients after choledochoscopic gallbladder-preserving surgery

move cholecystolithiasis and to preserve the function of the gallbladder, postoperative recurrence remains an issue. There are relatively few clinical studies on the recurrence of cholecystolithiasis after choledochoscopic gallbladder-preserving surgery. Lievens and Schutz et al. reported that the overall recurrence rate was $31 \%$ and that the cumulative probability was $44 \% 4$ years postoperatively in 100 patients after cholecystolithotomy [7]. The high recurrence rate was due to the improper selection of surgical indications, the use of old endoscopic techniques, and the lack of effective postoperative preventive measures. However, with the development of endoscopy techniques and the further recognition of gallbladder-preservation surgery, the recurrence rate of gallstones has decreased significantly. Tan et al. reported that the overall recurrence rate was
Table II. Univariate analysis of possible clinical factors for recurrence

\begin{tabular}{|c|c|}
\hline Variable & $P$-value \\
\hline Age $(<40 / \geq 40)$ & 0.765 \\
\hline Sex (male/female) & 0.514 \\
\hline $\operatorname{BMI}\left(<28 / \geq 28 \mathrm{~kg} / \mathrm{m}^{2}\right)$ & 0.192 \\
\hline Preoperative symptoms (yes/no) & 0.293 \\
\hline Pregnancy history $(\leq 1 / \geq 2)$ & 0.008 \\
\hline Preoperative TCH $(<5.17 / \geq 5.17 \mathrm{mmol} / \mathrm{l})$ & 0.529 \\
\hline Preoperative TG $(<1.7 / \geq 1.7 \mathrm{mmol} / \mathrm{l})$ & 0.803 \\
\hline Number of gallstones $(<3 / \geq 3)$ & 0.058 \\
\hline Maximal diameter of gallstones $(<2 / \geq 2 \mathrm{~cm})$ & 0.092 \\
\hline Gallbladder wall (normal/thickening) & 0.241 \\
\hline Gallbladder mucosa (smooth/rough) & 0.901 \\
\hline $\begin{array}{l}\text { Gallbladder contraction function } \\
(<30 \% / \geq 30 \%)\end{array}$ & 0.444 \\
\hline Uniformity of gallstones (uniform/nonuniform) & 0.002 \\
\hline Preoperative inflammation (present/absent) & 0.022 \\
\hline Postoperative oral medication (yes/no) & 0.022 \\
\hline Regularity of diet (regular/irregular) & 0.001 \\
\hline Family history (present/absent) & 0.068 \\
\hline Smoker (yes/no) & 0.127 \\
\hline Long-time drinking (yes/no) & 0.275 \\
\hline
\end{tabular}

TG - triglyceride, TCH - total cholesterol, BMI - body mass index.

4.92\% in 61 patients within a 26-month follow-up [8], and Gao et al. reported that the recurrence rate of 517 patients was $1.2 \%$, a very low recurrence rate, within a long-term follow-up [9]. Our study showed that the overall recurrence rate of cholecystolithiasis was $9.66 \%$ in 145 patients after choledochoscopic gallbladder-preserving surgery with a medi-

Table III. Results of multivariate analyses for recurrence after gallbladder-preserving cholecystolithotomy

\begin{tabular}{|lllc|}
\hline Variable & OR & $95 \% \mathrm{Cl}$ & $P$-value \\
\hline Pregnancy history $(\leq 1 / \geq 2)$ & 0.190 & $0.030-1.197$ & 0.077 \\
\hline Uniformity of gallstones (uniform/non-uniform) & 0.079 & $0.010-0.590$ & 0.013 \\
\hline Preoperative inflammation (present/absent) & 1.088 & $0.160-7.397$ & 0.931 \\
\hline Postoperative oral medication (yes/no) & 2.840 & $0.417-19.353$ & 0.286 \\
\hline Regularity of diet (regular/irregular) & 0.074 & $0.010-0.528$ & 0.009 \\
\hline
\end{tabular}

OR - odds ratio, $\mathrm{Cl}$ - confidence interval. 
A

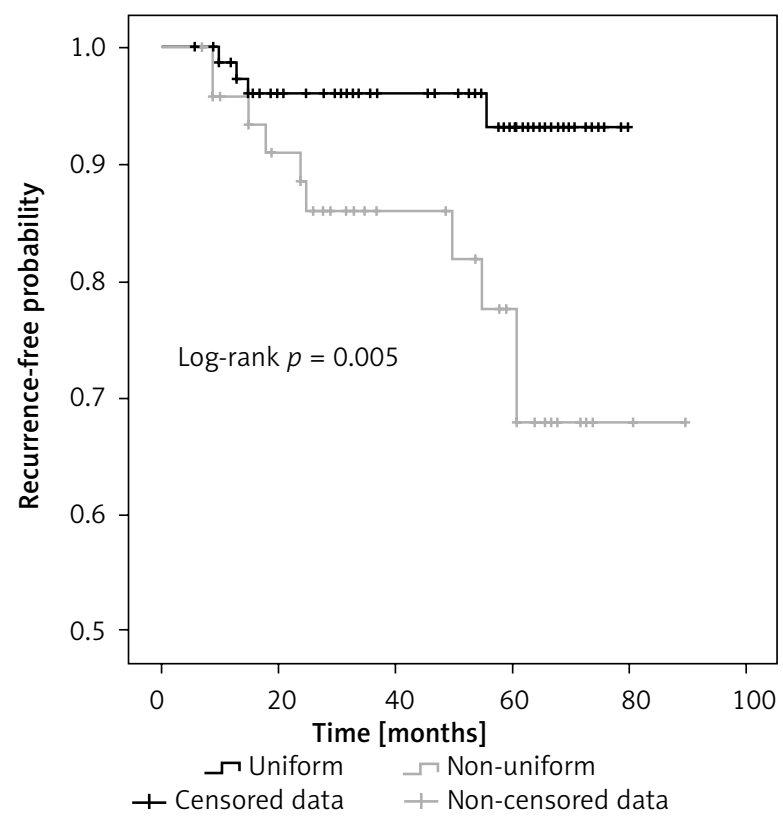

B

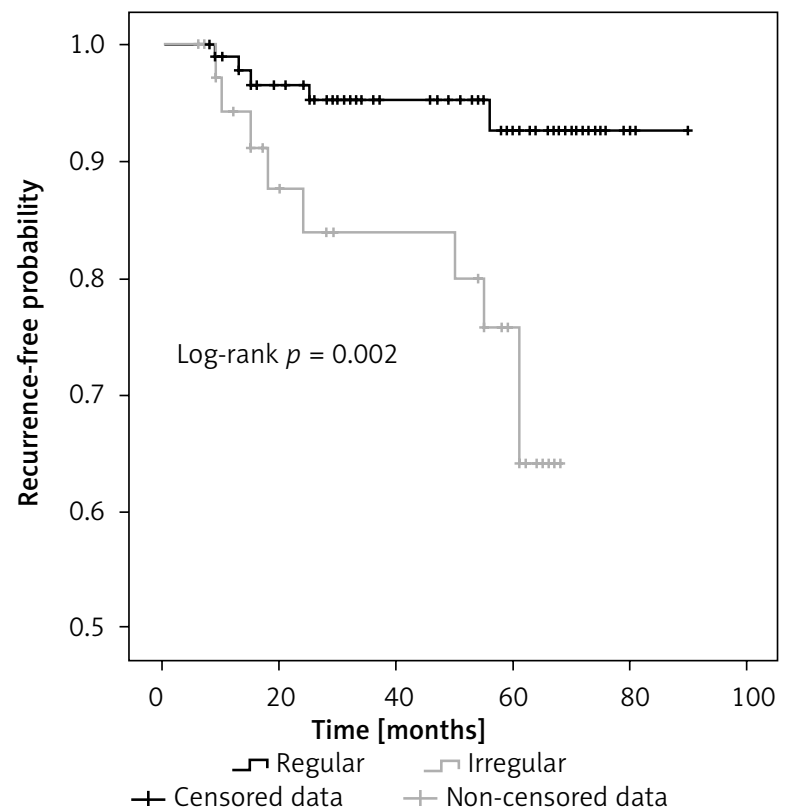

Figure 2. Kaplan-Meier analysis of the recurrence rate of patients with uniform gallstones versus nonuniform gallstones (A), regular lifestyle versus irregular lifestyle (B)

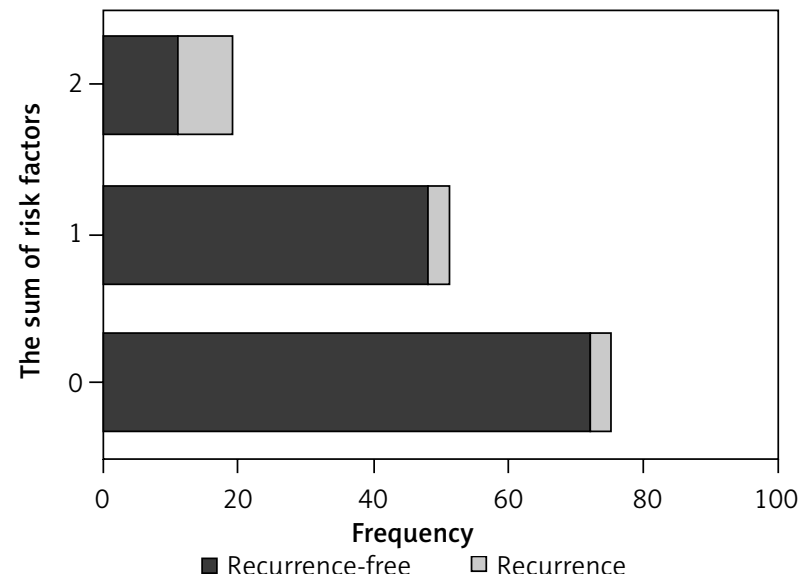

Figure 3. Number of patients with different independent risk factors

an follow-up of 36 months. There was a difference between the results of previous reports and those of our center. The reason for this difference may be the differences in the number of patients, in the length of follow-up, in the inclusion criteria and in the postoperative preventive measures. To explore the reason for postoperative gallstone recurrence, we speculated whether the reason for postoperative recurrence was related to high-risk factors for gallstone disease.
There was a sex difference, with a female prevalence, in the individuals in our study. The female-tomale ratio of the patients with recurrence was $2.5: 1$. However, the sex difference was not found to be related to recurrence, and the result is in line with a published study [26]. Gallbladder contraction function associated with recurrence for choledochoscopic gallbladder-preserving surgery has been reported. Huang et al. noted that the probability of stone recurrence after choledochoscopic gallbladder-preserving surgery was significantly higher in patients with poor gallbladder contraction function than in those with good gallbladder contraction function [27]. However, our study showed that gallbladder contraction function was not an independent risk factor for cholecystolithiasis recurrence. In China, it has been reported that gallbladder contraction function recovers significantly approximately 3 months after choledochoscopic gallbladder-preservation surgery in patients with poor gallbladder contraction function. The reason for this finding might be that the stimulation of the gallbladder mucosa was relieved after gallstones were removed. This discrepancy also might have occurred because the filled cholecystolithiasis or the obstructive cystic duct affected gallbladder emptying. Additionally, gallbladder contraction function is not equal to gallbladder 
function. Therefore, dissatisfactory gallbladder contraction function is not an absolute contraindication to choledochoscopic gallbladder-preserving surgery. Additionally, no statistically significant relationship was found in terms of age, BMI, pregnancy history, family history or recurrence. None of these factors should be considered indications or contraindications for this treatment.

There have been many reports about the relationship between the maximal diameter of gallstones or the number of gallstones and recurrence, but there has been no report about the relationship between the uniformity of gallstones and recurrence [26-28]. Villanova et al. found that patients with multiple stones had a significantly higher recurrence rate than those who had a single stone [29]. Tan et al. found that the probability of gallstone recurrence was significantly higher in patients with a maximal diameter of gallstones $\geq 2.0 \mathrm{~cm}$ [8]. However, our results showed that neither the maximal diameter of gallstones nor the number of gallstones was an independent risk factor for gallstone recurrence. Interestingly, the uniformity of gallstones was obviously related to gallstone recurrence. The difference in recurrence rate between the uniform gallstones and the nonuniform gallstones was also significant using Kaplan-Meier curve analysis after considering the recurrence time. These discrepant results might be because different stages of gallstone formation result in nonuniform gallstones. Therefore, patients with nonuniform gallstones should not be recommended to undergo choledochoscopic gallbladder-preserving surgery.

Eating patterns are currently recognized as a risk factor for gallstone formation. However, it was not conclusive whether recurrence after choledochoscopic gallbladder-preserving surgery was related to the regularity of diet. A regular eating pattern can decrease the cholesterol saturation of bile and can reduce the chances of cholestasis by promoting regular gallbladder emptying $[10,30]$. Excessive greasy meat intake can increase the probability of cholelithiasis; however, fruit, vegetable and nut intake can prevent cholelithiasis [14]. In our study, we discovered that the recurrence rate in patients with an irregular diet was higher than that in patients with a regular diet, and the difference was statistically significant. Therefore, a regular diet after choledochoscopic gallbladder-preserving surgery is crucial for preventing the recurrence of cholecysto- lithiasis. We encouraged patients to eat moderately fatty meals (vegetable fatty acid) early after surgery to promote gallbladder emptying.

The strength of this study was the mostly complete analysis of patients for recurrence of cholecystolithiasis after choledochoscopic gallbladder-preserving surgery with a long-term follow-up. The study also had some limitations. We were unable to obtain more specific factors, such as the shape and location of the gallstones, the components of the gallstones, and preoperative or postoperative differences. Further prospective studies and analyses with larger sample sizes are needed to confirm these clinical predictors.

\section{Conclusions}

Nonuniform gallstones combined with an irregular diet are significant risk factors that predict cholecystolithiasis recurrence. Choledochoscopic gallbladder-preserving surgery is not recommended for patients with nonuniform gallstones and an irregular diet. Furthermore, age, sex, BMI, pregnancy history, family history, postoperative oral drugs, gallbladder contraction function, maximal diameter of gallstones, number of gallstones, preoperative $\mathrm{TCH}$, and preoperative TG are not associated with gallstone recurrence.

\section{Conflict of interest}

The authors declare no conflict of interest.

\section{References}

1. Lammert F, Gurusamy K, Ko CW, et al. Gallstones. Der Gastroenterologe 2018; 13: 5-5

2. Ros E, Zambon D. Postcholecystectomy symptoms. A prospective study of gall stone patients before and two years after surgery. Gut 1987; 28: 1500-904.

3. McNamara DA, O'Donohoe MK, Horgan PG, et al. Symptoms of oesophageal reflux are more common following laparoscopic cholecystectomy than in a control population. Ir J Med Sci 1998; 167: 11-3.

4. Carrilho-Ribeiro L, Serra D, Pinto-Correia A, et al. Quality of life after cholecystectomy and after successful lithotripsy for gallbladder stones: a matched-pairs comparison. Eur J Gastroenterol Hepatol 2002; 14: 741-4.

5. Sand J, Pakkala S, Nordback I. Twenty to thirty year follow-up after cholecystectomy. Hepatogastroenterology 1996; 43: 534-7.

6. Kerlan RJ, LaBerge JM, Ring EJ. Percutaneous cholecystolithotomy: preliminary experience. Radiology 1985; 157: 653-6.

7. Lievens M, Schutz SM. Percutaneous cholecystolithotomy: is gall stone recurrence inevitable? Gastrointest Endosc 1995; 41: 271-2. 
8. Tan YY, Zhao G, Wang D, et al. A new strategy of minimally invasive surgery for cholecystolithiasis: calculi removal and gallbladder preservation. Dig Surg 2013; 30: 466-71.

9. Gao DK, Wei SH, Li W, et al. Totally laparoscopic gallbladder-preserving surgery: a minimally invasive and favorable approach for cholelithiasis. Exp Ther Med 2015; 9: 395-8.

10. Capron JP, Delamarre J, Herve MA, et al. Meal frequency and duration of overnight fast: a role in gall-stone formation? $\mathrm{Br}$ Med J 1981; 283: 1435.

11. Ortega RM, Fernández-Azuela M, Encinas-Sotillos A, et al. Differences in diet and food habits between patients with gallstones and controls. J Am Coll Nutr 1997; 16: 88-95.

12. Dyrszka H, Patel S, Sanghavi B, et al. Sonographic gallstone patterns are of value in predicting the outcome of biliary lithotripsy. Am J Gastroenterol 1991; 86: 1626-8.

13. Nayak L, Menias CO, Gayer G. Dropped gallstones: spectrum of imaging findings, complications and diagnostic pitfalls. $\mathrm{Br} J \mathrm{Ra}$ diol 2013; 86: 20120588.

14. Lammert F, Gurusamy K, Ko CW, et al. Gallstones. Nat Rev Dis Primers 2016; 2: 16024

15. Lin Y, Liu JN, Yong T, et al. Endoscopic minimal invasive cholecystolithotomy vs laparoscopic cholecystectomy in treatment of cholecystolithiasis in China: a meta-analysis. Int J Surg 2015; 13: 227-38.

16. Iranmanesh P, Frossard JL, Mugnier-Konrad B, et al. Initial cholecystectomy vs sequential common duct endoscopic assessment and subsequent cholecystectomy for suspected gallstone migration: a randomized clinical trial. JAMA 2014; 312 137-44.

17. Rong W, Liu J, Zeng Q, et al. Endoscopic minimally invasive gallbladder-preserving cholecystolithotomy and post-cholecystectomy syndrome. Chin J General Surg 2011; 20: 273-5.

18. Sowier S, Sowier A, Wiechowska-Kozłowska A, et al. Initial experience with endoscopic ultrasound-guided gallbladder drainage. Videosurgery Miniinv 2019; 14: 195-202.

19. Tan X, Wang G, Tang Y, et al. Minilaparoscopic versus single in cision cholecystectomy for the treatment of cholecystolithiasis: a meta-analysis and systematic review. BMC Surg 2017; 17: 91.

20. Sakorafas GH, Milingos D, Peros G. Asymptomatic cholelithiasis: is cholecystectomy really needed? A critical reappraisal 15 years after the introduction of laparoscopic cholecystectomy. Dig Dis Sci 2007; 52: 1313-25.

21. Wei S. The clinical application of the hard gallbladder endoscope combined with soft choledochoscope in the surgery of laparoscopic microscopic trauma for the removal of calculi and preservation of gallbladder. J Laparoendosc Adv 2013; 23: 106-8.

22. Pal AK, Singh D, Sonkar AA, et al. Re: Single-incision versus 3-port laparoscopic cholecystectomy in symptomatic gallstones: a prospective randomized study. Surgery 2017; 162: 1347.

23. Zhang Y, Peng J, Li X, et al. Endoscopic-laparoscopic cholecystolithotomy in treatment of cholecystolithiasis compared with traditional laparoscopic cholecystectomy. Surg Laparosc Endosc Percutan Tech 2016; 26: 377-80.

24. Matayoshi N, Sato N, Okimoto T, et al. A review of 7 cases of laparoscopic cholecystectomy for pediatric cholecystolithiasis. J Uoeh 2017; 39: 223-7.
25. Zha Y, Chen XR, Luo D, et al. The prevention of major bile duct injures in laparoscopic cholecystectomy: the experience with 13,000 patients in a single center. Surg Laparosc Endosc Percutan Tech 2010; 20: 378-83.

26. Li QF, Xu X, Ge X. Gallstone recurrence after minimally-invasive cholecystolithotomy with gallbladder reservation: a follow-up of 720 cases. Eur Rev Med Pharmacol Sci 2015; 19: 1403-6.

27. Huang H, Zou YP, Du JD, et al. Gallstone recurrence after successful percutaneous cholecystolithotomy:a 10-year follow-up of 439 cases. Hepatobiliary Pancreat Dis Int 2007; 6: 199-203.

28. Wang T, Luo H, Yan HT, et al. Risk factors for gallbladder contractility after cholecystolithotomy in elderly high-risk surgical patients. Clin Interv Aging 2017; 12: 129-36.

29. Villanova N, Bazzoli F, Taroni F, et al. Gallstone recurrence after successful oral bile acid treatment. A 12-year follow-up study and evaluation of long-term postdissolution treatment. Gastroenterology 1989; 97: 726-31.

30. Ortega RM, Fernández-Azuela M, Encinas-Sotillos A, et al. Differences in diet and food habits between patients with gallstones and controls. J Am Coll Nutr 1997; 16: 88-95.

Received: 1.06.2019, accepted: 27.07.2019. 